\title{
Modeling of vibration transmission through impact elements
}

\author{
Vitaly Krupenin \\ Mechanical Engineering Research Institute of the Russian Academy of Sciences, Moscow, Russian Federation
}

\begin{abstract}
Rod waveguides are important technical objects that serve to transmit vibrations between machine elements. Such a transfer is determined by their design features and can be both desirable and harmful. The article deals with the problem of modeling the propagation of a monoharmonic wave through a rod waveguide, in which a discontinuity appeared, and a shock pair was formed. The features of the formation of polyharmonic waves and manifested nonlinear effects leading to a fundamental distortion of the dynamic pattern are described. The method of time-frequency analysis of vibration impact processes, which determine the dynamics of the system, is used. A calculation scheme and examples are given.
\end{abstract}

\section{Introduction}

The purpose of this article is to provide methods for calculating and analyzing systems that transmit vibrations through rod systems containing single isolated impact pairs. The problems associated with the description of the vibration fields arising from the propagation of elastic waves in the constructions of machines modeled by such systems seem to be relevant and have a large number of specific implementations. Very often, impact pairs occur due to some faults, but, of course, they can correspond to the design features of the systems. For example, in ultrasonic technological machines, the workflow is determined by the systematic collisions of the instrument with the medium being processed, and the necessary energy is transferred using rod waveguides. In any case, the ability to consider the peculiarities caused by heterogeneity and the formation of impact pairs is an interesting and multifaceted task. Many studies have been devoted to different approaches to its study, among which we note, for example, [1-9].

\section{Typical models}

An example of a linear waveguide with point-like inclusions in the form of a system of solids is shown in Fig. 1. This system is well studied $[2,10]$.

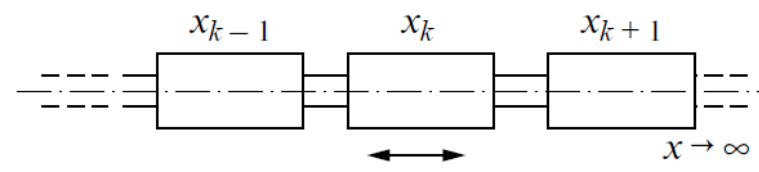

Fig. 1. Linear rod with point inclusions.

Assuming the rod is infinite, and the point inclusions are the same (all $m_{k}=m$ ), with coordinates $x_{k}$, denoting, $E, S, \rho-$ the Young's modulus, cross-sectional area and linear density of the rod, one can obtain the equation of motion in partial derivatives:

$$
u_{t t}-E S \rho^{-1} u_{x x}=\rho^{-1} f(x, t)-m \rho^{-1} \sum_{(\mathrm{k})} u_{t t}\left(x_{k}, t\right),
$$

Here $\rho^{-1} f(x, t)$ is the distribution of external influences. Without limiting generality, we can further assume that $\rho=1$.

Suppose, for some reason, there have been any structural changes or violations of power closures. Then at the place of inclusions marked in Fig. 1, various shock pairs (discontinuities) arise. An example for an isolated impact pair with a coordinate $x=0$ is given in Fig. 2 . The discontinuity of the system is determined by the "external limiter". Equation (1) is transformed into a nonlinear equation

$$
u_{t t}-c^{2} u_{x x}=f(x, t)-\Phi_{0}\left[u(t, x), u_{t}(t, x)\right] \delta(x)
$$

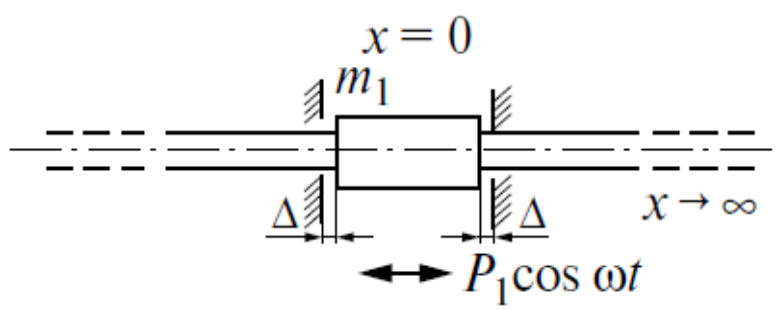

Fig. 2. External limiter.

Here $c=\sqrt{E S}$ - is the speed of sound in the material of the rod; $\delta(x)$ - Dirac function; external force is also reduced to a point $x=0 ; \Phi_{0}$ - the force of the shock interaction, corresponding to any hypothesis of impact, for example, the hypothesis of Newton [2]. Therefore,

\footnotetext{
*Corresponding author: krupeninster@gmail.com
} 
for the system Fig. 2, you can write the terms of the impact in the form

$$
J_{\alpha}=(1+R) m\left|u_{t}\left(t_{\alpha}-0,0\right)\right| ; u\left(t_{\alpha}, 0\right)=\Delta ;|u(t, x)|<\Delta .
$$

Here $\left(J_{\alpha}, t_{\alpha}\right)$-is the impulse and moment of $\alpha$-th impact; 2 $\Delta$-gap size (Fig. 2); $R \in(0,1]$ - restitution coefficient. In this case $\Phi_{0}=J_{\alpha} \delta\left(t-t_{\alpha}\right)$. In the case of alternate periodic collisions with each of the limiters (Fig. 2): $\Phi_{0}=J \delta^{\frac{T}{2}}\left(t-t_{0}\right)$, where, due to the periodicity, the impulses are the same $\left(J_{\alpha} \equiv J\right)$, and the phase $t_{0}$ is fixed, since the impacts are repeated every half of the period T. Generalized periodic symmetric function

$$
\delta^{\frac{T}{2}}(t)=\sum_{j=-\infty}^{\infty}\left[\delta(t-j T)-\delta\left(t-j T-\frac{1}{2} T\right)\right]
$$

Our goal is to describe the process of passing a monoharmonic wave, excited by force $f(x, t)=P_{1} \delta(x) \cos \omega t$, through an impact pair.

\section{Periodic modes}

Further, we confine ourselves to the most important for the tasks of this kind of periodic modes.

If forces with a distribution $g\left(x, t, u, u_{t}, \cdots\right)$ act on the rod, then an operator relationship takes place [2]:

$$
u(x, t)=\int_{D} L(p, x, y) g\left(x, y, u, u_{t}, \cdots\right) d y ; p \equiv \frac{\partial}{\partial t},
$$

$\mathrm{D}$ is the set on which the function is defined. Here the problem of wave propagation is considered and assumed that $-\infty<x<\infty$. Function $L(p, x, y)-$ describes the dynamic compliance operator. For this system $[2,6,8$, 9]:

$$
\begin{aligned}
& L(x, y, p)=p^{-1} l(p, x, y)-\frac{m p l(2 p, x, y)}{1+m p l(p, x, y)}, \\
& l(p, x, y)=\frac{1}{2 c} \exp \left[-\frac{p}{c}|x-y|\right] .
\end{aligned}
$$

Representation (5) turns into an equation if, among the arguments of a complicated function $g$, there are functions that need to be defined. Thus, the solution of equation (2) will consist of the sum of the solution of the linear problem and the function depending on the impact parameters to be determined. In accordance with the methods of time-frequency analysis of vibro-impact processes $[2,6]$, you can get the following presentation:

$$
u(x, t)=P_{1}|L(x, 0, i \omega)| \cos [\omega t+\psi(x)+\varphi]-J \chi(t, x) .
$$

Formula (7) is determined using the following quantities and functions. (i) The modulus and argument of dynamic compliance from the point of application of an external force to an arbitrary point $x$ :
$|L(x, 0, i \omega)|$ и $\psi(x)$. (ii) Phase shift, assuming that the moment of impact is combined with the beginning of time времени, $\varphi \in[0, T / 2]$. (iii) $\chi(t, x)$ - reaction of a linear system (rod) to a force action of the form (4) $\delta^{\frac{T}{2}}(t)$.

A function $\chi$ is called a symmetric periodic Green function. The theory of such functions is well developed [2]. In this case

$$
\chi(t, x)=\frac{2}{\mathrm{~T}} \sum_{k=-\infty}^{\infty} L[x, 0, i(2 k+1) \omega] \exp [i(2 k+1) \omega t] .
$$

That is, the Fourier coefficients are determined using relations (6). There are ways to record the series (8) in the form does not require infinite series for $t \in[0, T / 2]$. Thus, the task of finding the periodic modes of this type has been reduced to finding the unknown motion parameters that determine the representation (7). This can be done using impact conditions (3), replacing $t_{\alpha} \equiv \varphi, J_{\alpha} \equiv J$ and considering that collisions occur at $\mathrm{x}$ $=0$.

\section{Analytical solution}

In order to obtain an exact analytical solution of the form (7), it is necessary to carry out transformations, which we mainly omit here, showing the most significant results.

With the help of formula (6) we can find

$L(x, 0, p)=\frac{D(p, x)}{2 p c+m_{1} p^{2} D(p, x)} ; D=\exp \left[-\frac{p}{c}|x|\right]$.

Now with the help of (8) for $p=i(2 k+1) \omega$ you can define the function $\chi(t, x)$. For $x=0$ : $W(p)=\left(2 p c+m_{1} p^{2}\right)^{-1} ;|W(p)|=\left[\sqrt{m_{1}^{2} \omega^{4}+4 \omega^{2} c^{2}}\right]^{-1} ;$ $|W(i \omega)|=\left[\sqrt{m_{1}^{2} \omega^{4}+4 \omega^{2} c^{2}}\right]^{-1} ; W(i \omega) \equiv L(0,0, i \omega) ;$ $\arg L(0,0, i \omega)=\operatorname{arctg}\left(2 c / m_{1} \omega\right)$. Further we will designate $|L(0,0, i \omega)| \equiv U_{0}$. From the relation (7) for the point $x=0$ we get

$$
u(x, t)=P_{1} U_{0} \cos (\omega t+\gamma)-J \chi(t, 0),
$$

where the motion parameters - impulse and impact phase $(J, \gamma)$ - can be found from the impact conditions (3). At the same time, it is necessary to take into account the periodicity of the process and combine the impact with the beginning of the counting of time. We have:

$$
\begin{gathered}
P_{1} U_{0} \cos \gamma=\Delta-J \chi(0,0) \\
P_{1} U_{0} \omega \sin \gamma=-J\left[\frac{\partial \chi(-0,0)}{\partial \mathrm{t}}+\frac{1}{(1+R) m_{1}}\right] \equiv-J Z_{0},
\end{gathered}
$$


where the physical meaning of the value $Z_{0}$ entered is obvious: it describes the loss of energy on impact. Excluding the phase from (11), (12), we obtain two values of the impulse under sinusoidal excitation:

$$
J_{1,2}=\frac{-\Delta \chi_{0} \pm \sqrt{P_{1}^{2} U_{0}^{2} \chi_{0}^{2}-\omega^{-2} Z_{0}^{2}\left(\Delta^{2}-P_{1}^{2} U_{0}^{2}\right)}}{\chi_{0}^{2}+\omega^{-2} Z_{0}^{2}},
$$

where $\chi_{0}=\chi(0,0)$. Using numerical methods, it was found that the plus sign in front of the radical corresponds to a stable solution. Therefore, we will discard the modes corresponding to the minus sign. Taking into account the computed, we obtained all the relations defining formula (10). In particular

$$
\chi(t, 0)=\frac{2}{\mathrm{~T}} \sum_{k=-\infty}^{\infty} \frac{\exp [-i(2 k+1) \omega t]}{2 i(2 k+1) \omega c-m_{1}(2 k+1)^{2} \omega^{2}} .
$$

On the symmetry interval using the formulas given in [2], series (14) can be written in a compact form. For $0 \leq t<T / 2$ :

$$
\begin{gathered}
\chi(t, 0)=\frac{1}{4 c}-\frac{1}{2 c} \frac{\exp \left(-2 c t \mathrm{~m}_{1}^{-1}\right)}{1+\exp \left(-c T m_{1}^{-1}\right)} ; \chi(0,0)=-\frac{1}{4 c} \text { th } \frac{c T}{2 m_{1}} ; \\
\frac{\partial \chi(-0,0)}{\partial \mathrm{t}}=\frac{1}{m_{1}} \frac{1}{1+\exp \left(-c T m_{1}^{-1}\right)} .
\end{gathered}
$$

The condition for the existence of a periodic vibroimpact process follows from the conditions of the realizability of relations (11) - (13). From the second relation (12) we find, taking into account the relations found, the condition of existence in the form:

$$
\left|\frac{J_{1} \sqrt{m_{1}^{2} \omega^{4}+4 c^{2} \omega^{2}}}{P_{1} \omega m_{1}}\left[\frac{1}{1+\exp \left(-c T m_{1}^{-1}\right)}+\frac{1}{(1+R)}\right]\right| \leq 1 .
$$

Due to the presence of wave resistance in an infinite $\operatorname{rod}[11,12]$, the value of $\operatorname{Im} L(x, y, i \omega) \neq 0$ and vibroimpact mode at low levels of excitation ceases to exist even in the absence of energy loss during impact $(\mathrm{R}=0)$. The nature of dependence $J(\omega)$ is shown in Fig.4. The dashed line corresponds to unstable modes $\left(J_{2}\right)$.

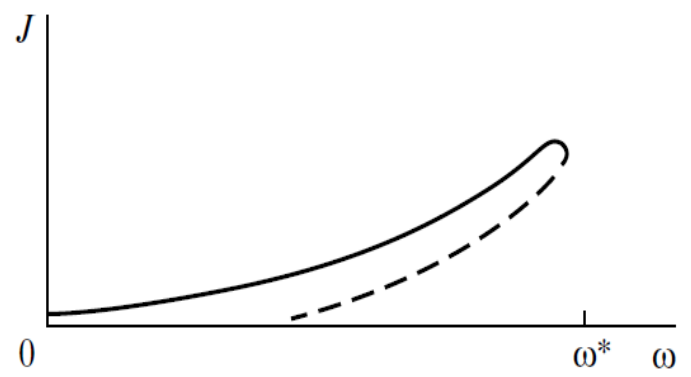

Fig. 3. The dependence of the impact impulse on the frequency.

It can be seen that this dependence is in many ways like the nonlinear resonance dependencies found in the traditional theory of vibro-impact systems $[2,12]$. The boundary frequency $\omega^{*}$ is determined by the sign of equality in the last inequality and depends on the resistance to motion. Near the frequency $\omega=0$, the modes of the considered type do not exist.

Before reaching the frequency $\omega=\omega^{*}$, collisions are intense. Waveguide is in a resonant state. After the passage of this frequency $\left(\omega>\omega^{*}\right)$ processes with intense collisions are impossible. For a rod with limiters, nonlinear effects attached to traditional vibro-impact systems can be observed. We note some of them $[2,6$, 12].

(I) The dynamic phenomena of frequency and amplitude pulling. You can get intense vibro-impact modes by gradually increasing the excitation frequency (the phenomenon of pulling in frequency). The impact pulse is proportional to the size of the gap. By smoothly increasing the gap, it is possible to achieve an increase in the amplitude of oscillations and the intensity of the vibroimpact mode (the phenomenon of pulling in amplitude).

(II) Downward jump and hard start. As noted, after passing through the boundary conditions for the existence of resonant regimes, a sudden decrease in the amplitude of the oscillations occurs -"downward jump". Return to resonant vibroimpact modes is possible only after additional impact at a frequency that is lower than the frequency of downward jump (the possibility of a hard start). Return without a hard start is possible only after a significant decrease in the frequency value.

The process of wave propagation in the waveguide describes the representation (7). An analysis of this representation shows that a longitudinal wave propagates in the system along the rod to both sides of the included shock pair. This wave contains a complete set of harmonic components of multiples of the exciting frequency. In this case, it is possible such a combination of system parameters that the propagating waves can contain only higher harmonic components. Waveguide will be locked for the main tone.

When dissipative factors are introduced into the model, in addition to energy dissipation upon impact, each of the harmonic components of the wave will exponentially decay (as $\exp (-\alpha x), \alpha>0$.

\section{Other models}

Similarly, other waveguide models with discontinuities can be studied. Examples of waveguides with internal discontinuities are given in Fig.4, 5.

Fig. 3 shows a symmetrical impact pair located inside the rod. The equation of motion of type (2) is added to the equation of the relative motion of the striker in the cavity.

In Fig. 5 impact pair - asymmetrical; an impact oscillator is placed inside the rod $[2,10]$.

Methods for analyzing data systems are similar to those above. Here the impact conditions are recorded for relative coordinates and speeds.

The list of waveguide models with discontinuities can be significantly extended $[6,7]$. 


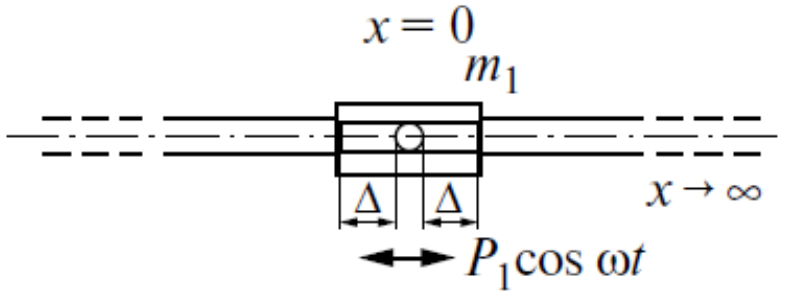

Fig. 4. The waveguide with the internal discontinuity (I).

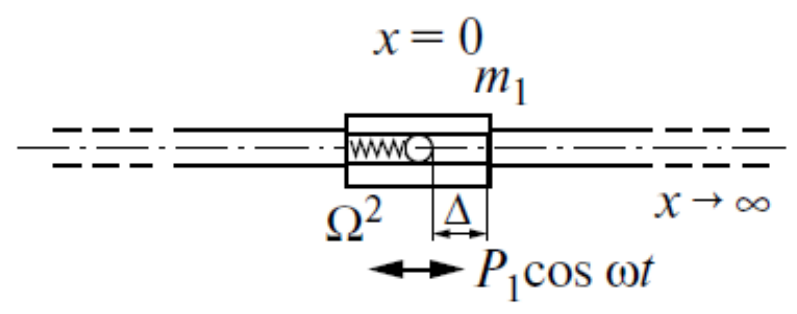

Fig. 5. The waveguide with the internal discontinuity (II).

For example, in Fig. 6 shows a model of two colliding rods with attached percussionists.

Such a model [12] can be used to study the dynamics of ultrasonic technological machines.

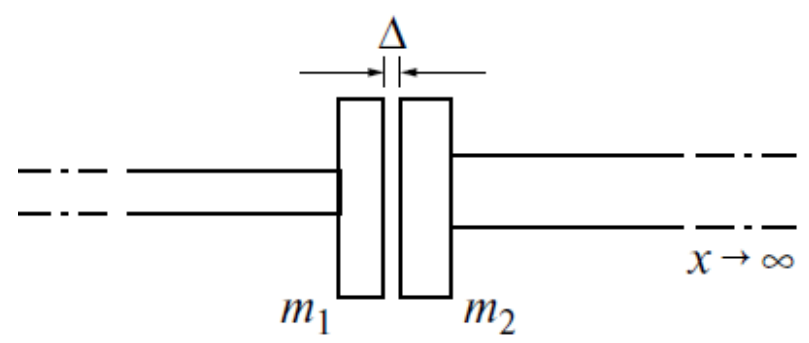

Fig. 6. Impacting semi-infinite rods with percussionists.

\section{Concluding comments}

We note, first, that if we reject the assumption of instantaneous collisions, we can apply the singularization method and use more accurate impact theories [13].

Secondly, the analysis of subharmonic, ultraharmonic and combination vibro-impact modes and the corresponding waves, arising, for example, under the action of a periodic polyharmonic external force, is carried out in a completely similar way $[5,12]$.

Any significant additional assumptions entail, in turn, complication of the necessary calculations and, quite often, lead to the loss of the opportunity to obtain approximate analytical expressions, similar to those obtained above. At the same time, the proposed method, of course, is easily combined with numerical methods and can help achieve the required accuracy of solutions $[11,14]$.

Thirdly, the specified complications concern also structures of the considered objects. The used method of time-frequency analysis of vibro-impact processes allows us to consider, for example, composite rods or multidimensional core and beam structures, etc.

Here, it is important to know the corresponding dynamic compliance operators, which determine the periodic Green functions necessary for obtaining solutions.

\section{Conclusions}

Strongly nonlinear factors, which manifest themselves in waveguides due to the formation of shock pairs through which the waves pass, not only introduce a distortion of their appearance, but also cause specific dynamic effects. As a result, the waves become polyharmonic. Their passage is accompanied by the development of nonlinear resonance phenomena and the appearance of intense vibro-impact modes near certain frequencies. The method of time-frequency analysis of vibro-impact processes allows us to give an adequate description of the systems of this type.

The paper was supported by the Russian Foundation for Basic Research (project \# 18-08-00168).

\section{References}

1. A. Veprik, P. Voznyuk, V. Krupenin, I. Chirkov Broadband Vibro-Impact Generators of Mechanical Oscillations (Mashinostroenie, Leningrad, 1987)

2. V. Babitsky, V. Krupenin Vibration of Strongly Nonlinear Discontinuous Systems (Springer, 2001)

3. S. Lepri, A. Pikovsky CHAOS, 24, p. 043119. http://dx.doi.org/ doi 10.1063/1.4899205 (2014)

4. R. Ibrahim Vibro-Impact Dynamics (Springer, 2009)

5. V. Krupenin Dokl. Akad. Nauk, 343, 6 (1995)

6. V. Krupenin J. Mach. Manuf. Reliab., 47, 2, (2018)

7. V. Krupenin J. Mach. Manuf. Reliab 45, 4 (2016)

8. I. Artobolevsky, Yu. Bobrovnitsky, M. Genkin Introduction to Acoustic Dynamics of Machines (Nauka, Moscow, 1979)

9. A. Butkovsky Characteristics of DistributedParameter System (Nauka, Moscow, 1979)

10. V. Burd, V. Krupenin Averaging in QuasiConservative Systems (Belyi Veter, Moscow, 2016)

11. V. Astashev, V. Krupenin Probl. Mashinostr. Nadezhnosti Mash., 5 (1998)

12. V. Astashev, V. Krupenin Non-linear Dynamics of Ultrasonic Technological Processes (MGUP im Ivana Fedorova, Moscow, 2016)

13. V. Krupenin J. Mach. Manuf. Reliab., 45, 2, (2016)

14. V. Krupenin J. Mach. Manuf. Reliab. 39, 1 (2010) 